\section{Faserforschung und}

WISSENSCHAFTLICH-TECHNISCHE ZEITSCHRIFT FUR DIE CHEMIEFASER- UND TEXTILINDUSTRIE

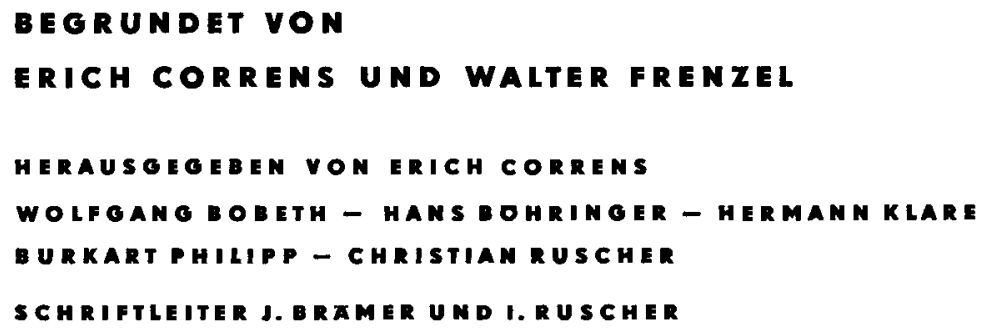

\author{
AUS DEM INHALT \\ Reinisch und Ulrich \\ Zur Reaktion von Polycaprolactam \\ mit Formaldehyd \\ Teil II. Analytische Bestimmung der \\ Hydroxymethyl- und Methylensubstitution \\ Runge und Nelles \\ Zur Inhibierung der thermischen Verfärbung \\ des Polyacrylnitrils \\ Philipp und Alsleben \\ Über Symplexe von Cellulosederivaten \\ V. Mitt.: Untersuchungen zur \\ Leitfähigkeitstitration anionischer \\ Cellulosederivate mit polymeren Aminen \\ Dautzenberg \\ Lichtstreuung an mäßig konzentrierten \\ Polystyrollösungen \\ Teil I: Einfluß der relativen Molekülmasse \\ Kudrna \\ Kaltreckung von linearen Polymeren \\ Jahreskolloquien des Institutes \\ für Textiltechnologie der Chemiefasern \\ Rudolstadt 1969 \\ Neue Bücher \\ Patentschau \\ Literaturschau
}




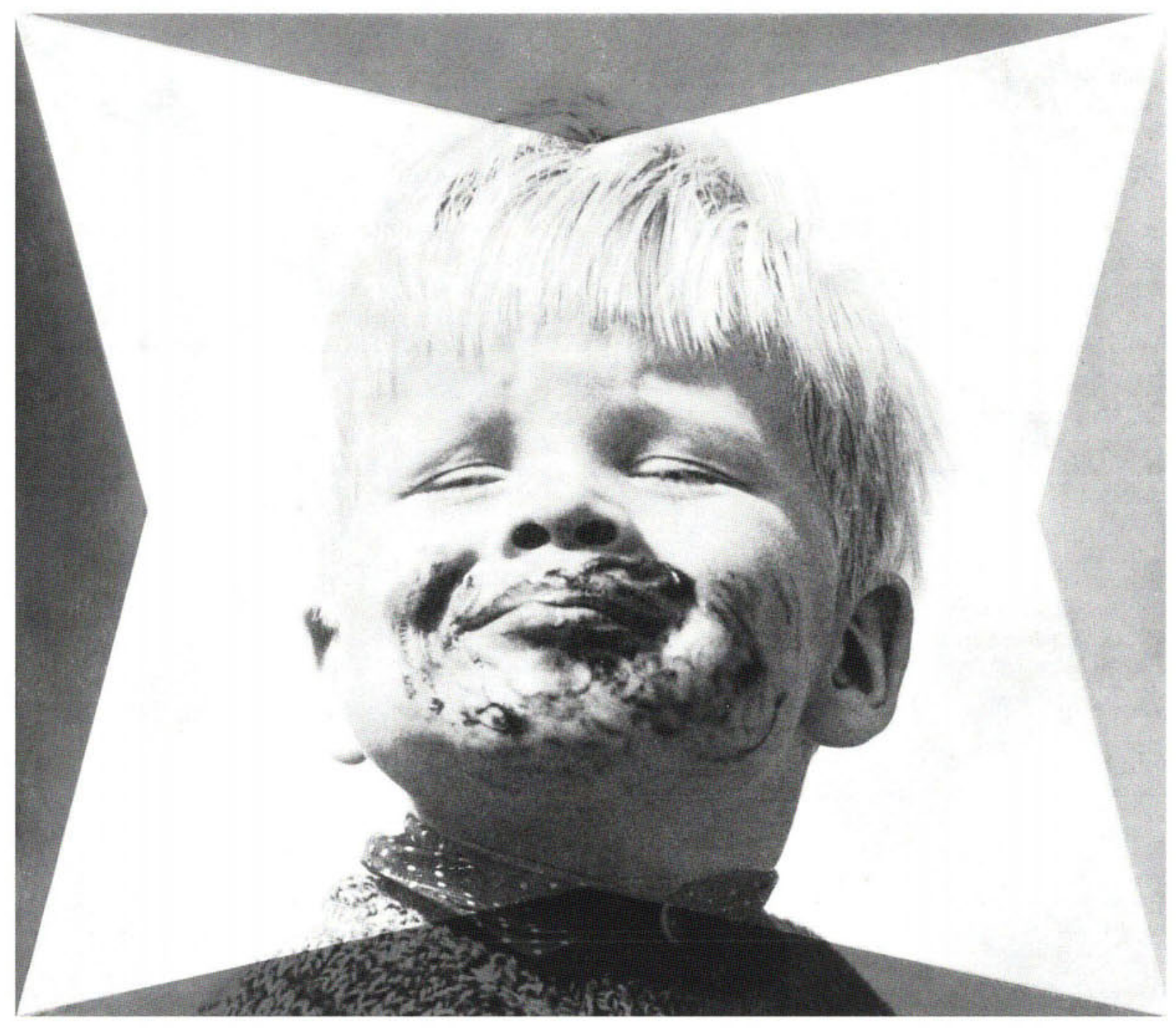

Hier genügt Seife!

Die Textilindustrie braucht jedoch geeignete Waschmittel, die den unterschiedlichen Verschmutzungen sowie den variablen

Materialzusammensetzungen Rechnung tragen.

Waschmittel aus Schkopau - unter dem Namen

PRA'WOZELL ${ }^{\circledR}$ - werden durch ihre besonderen

Vorzüge, wie Härtebeständigkeit - gutes

Netzvermögen - Metallsalz- und Chemikalien-

beständigkeit - hohe Entfettungskraft - niedrige

Anwendungstemperaturen und Anwendungs-

konzentrationen - keine Faserschädigungen sowie

gute Gleitwirkung beim Walken, den Anforderungen

der Textilindustrie unserer Zeit gerecht.

Fordern Sie Informationsmaterial an.

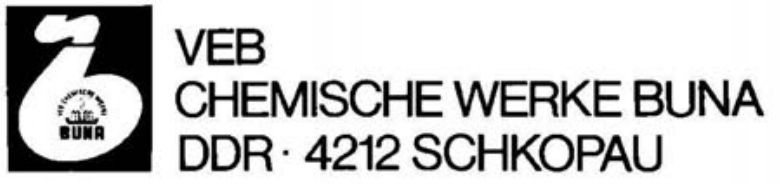




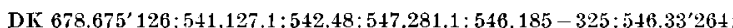
$546.32^{\prime} 267: 543.2+2$

Reinisch, Gerhard, und Ulrich, Mans-IIeinz

Zur Reaktion von Polycaprolactam mil. Formaldehad

Teil II. Analytische Bestimmung der IJydroxymethyl- und Melliylensubstitution

Faserforsch. u. Textiltcchnik 21 (1970) 3, S. 101-105. 13 Tab., 8 Lit.

Analysenmethoden für formaldehydmodifiziertes Polycaprolactam werden beschrieben und benteilt. Die Abspaltung des Hydroxymethylgruppenformaldehyds crfolgt durch $2 \mathrm{~h}$ Destillation unter Zusatz von $2 \mathrm{n}$ Natriumcarbonatosing oder durch hnmittelbare Linwirkung alkalscher KCNgruppen gebundene Formaldehyd wird nach der Hydroxymothyluppenabspaltung durch Destillation inter Zusatz halbkonz. Phosphorsâre freigesetzt.

DK 678.745.32: [677.019.17:541.12.036]: 541.128.4:542.953:547.462.3'312: $547.462 .3^{\prime} 0541$

Runge, Jürgen, und Nelles, Wally

Zur Inhibierung der thermischen Verfärbung des Polyaerylnitrils

Faserforseh. u. Textiltechnik 21 (1970) 3, S. 105-110. 3 Abb., 4 Formelbilder, 4 Tab., 18 Lit.

Die Verfärbung, die Polyacrylnitril im Temperaturbereich von 120 bis $220^{\circ} \mathrm{C}$ crleidet, wird, soweit sie auf die nucleophil initiierte Kondensation durch Maleinimid und dessen N-Substitutionsprodukte wirksani inhibiert.

DK 678.546.11:547.458.82:543.257:541.135:678.74:541.6:541.24

Philipp, Burkart, und Alsleben, Horst

Uber Symplexe von Cellulosederivaten

V. Mitt.: Untersuchungen zur Isitfahigkeitstitration anionischer Cellulosederivate mit polymeren Aminen

Faserforsch. u. Textiltechnik 21 (1970) 2, S. 110-116. 7 Abb., 3 Tab., 22 Lit.

Dic Symplexbildung der Polyelektrolyte Natriumearhoxymethylerllulose und Natriumeellulosexanthogenat mit quaternisierten Polyvinylverbindungen in wäßriger Lösung läßt sich mit Hilfe der Leitfähigkeitstitration verfolgen. Der K nickpunkt der Titrationskurve cutspricht, unabhängig von einem aquivalenten Verhältnis der Polyionen, sofern diese in Salze eingesetzt werden.
DK $678.5 / .8 .027 .42: 677.494 .021 .64: 677.494 .021 .97: 016$

Kudrna, Miloslav

Kaltreckung von linearen Polymeren

Faserforsch. u. Textiluchnik 21 (1970) 3, S. 125-13/. $9 \mathrm{Abb}, 56 \mathrm{Lit}$

Die hauptsächlichsten Theorien und Vorstellungen über den Mechanismus der Kaltreckung yon Polymercn werden anhand der Literatur beschrieben.

УК 061.3.055.1,,1969“":677.4:001.891(430.2)

Jahreskolloquien des Institutes für Textiltechnologic der Chemiclasern Rudolstadt 1969

Faserforsch. u. Textiltechnik $21(1970)$ 3, S. 134-136.

Neue Büicher S. $136-138$.

Patentschau S. $138-1 / 1$.

Literalurschau S. 1/11-14'1.

DK 678.746.22:541.24:541.8:535.36:539.2

Dautzenberg, Herbert

lichtstreuung an mäßig konzentrierten Polystyrollösungen Teil I, : Einfluß der relativen Holekülmasse

Faserforsch, u. Textiltechnik 21 (1970) 3, S. 117-12/. 13. $\Lambda$ bb., 2 Tab., 9 Lit.

Zur Vntersuchung des Lösungszustandes im Bereich höherer Konzentrit tionen wurden die streufunktionen yon Polystyrolfraktionen mit relativen Molekülmassen von 1,3 bis $7,1 \cdot 10^{8}$ in Abhängigkeit von der Konzentration aufgenommen. Der Konzentrationsbereich erstreckte sich yon 0,02 bis $1 \%$. Aus der Maximumshedingung der Funktion $I(c)$ wurden die dritten Virialkoeffizienten bestimmt. Besondere Aufmerksamkeit wurde dem Problem geschenkt, bis zu welchen Konzentrationen die Streukurven durch die struktur der Hinzelmolekule bestimmt worlen und ab wana eine Netz- 


\title{
Grundlagen der Polarographie
}

\author{
Von JAROSLAV HEYROVSKY Und JAROSLAV KUTA \\ (Übersetzung aus dem Tschechischen) \\ In deutscher Sprache herausgegeben von KURT SCHWABE \\ unter Mitarbeit von HANS JOACHIM BÄR
}

1965. XVII, 562 Seiten - 256 Abbildungen - 19 Tabellen - gr. $8^{\circ}$ - Leinen M 73,-

Viele Jahre war das 1941 herausgegebene, von J. Heyrovsky verfaßte Buch „Polarographie“ das Standardwerk und eine unentbehrliche Informationsquelle für dieses Spezialgebiet. Wenn jetzt die deutsche Übersetzung der von J. Heyrovský und seinem Mitarbeiter J. Küta besorgten tschechischsprachigen Neubearbeitung erscheint, dürfte damit ein langgehegter Wunsch erfüllt sein.

Dieses Werk vermittelt eine Übersicht über die theoretischen Ergebnisse der Polarographie in ihrer vierzigjährigen Entwicklung und zeigt die Fortschritte auf dem Gebiet der Polarographie auf, namentlich die neuen und verbesserten Theorien aller Arten von polarographischen Strömen und der entsprechenden Elektrodenprozesse. Das polarographische Verhalten anorganischer und organischer Stoffe und die praktische Anwendung der Polarographie blieben unberücksichtigt, da über diese Komplexe bereits genügend Fachliteratur vorhanden ist. Die Methodik geht von experimentell beobachteten Erscheinungen aus, die theoretisch leicht zu begründen sind. Die mathematische Formulierung und die Deutung der ihrer physikalisch-chemischen Natur entsprechenden Erscheinungen ist ein wichtiges Anliegen dieses Werkes. Das neue und umfangreiche Buch, in dem naturgemäß die von Heyrovský und seiner Schule entwickelten Verfahren eine besonders ausführliche Behandlung erfahren, dürfte ebenso wohlwollend aufgenommen und eifrig benutzt werden wie die altbewährte "Polarographie“.

Bestellungen durch eine Buchhandlung erbeten

A K A D E M I E - V E R L A G B ERL I N

Die Zeitschrift ,Faserforschung und Textiltechnik“ erscheint monatlich in Heften zu 48 Textseiten im Format A 4. Der Preis für das Einzelheft beträgt M 15,- (Sonderpreis für DDR M 9.-), für den Vierteljahrbezug M 45, - (Sonderpreis für DDR M 27,-), zuzügl. Bestellgeld. Die Berechnung erfolgt zu Beginn eines Vierteljahrs für 3 Hefte. Bestellungen aus dem Gebiet der Deutschen Demokratischen Republik an ein Postamt, eine Buchhandlung oder den straße 4-6, aus dem Ausland an eine Importbuchhandlung, den Deutschen Buch-Export und -Import GmbH.. 701 Leipzig, PostschlieBfach 276, oder den straße 4-6, aus dem Ausland an eine Importbuchhandlung, den Deutschen Buch-Export und -Import GmbH.. 701 Leipzig, PostschlieBfach 276, oder den
Akademic-Verlag GmbH., 108 Berlin, Leipziger Str. 3-4 (Fernruf: 220441; Telex-Nr. 011 2020: Postscheckkonto 35021) erbeten. Bestellnummer dieses Akftes: 1014/21/3. Alleinige Anzeigenannahme DEWAG-WERBUNG, 102 Berlin, Rosenthaler Str. 28/31, und alle DEWAG-Betriebe in den Bezirksstadten der DDR. - Bestellungen in der UdSSR nehmen entgegen: Städtische Abteilungen von „SOJUZPECHATJ" bzw. Postămter und Postkontore.

Herausgeber und verantwortlich für den Inhalt: Prof. Dr. Dr. Erich Correns, Institut für Faserstoff-Forschung der DeutschenAkademie der Wissenschaften zu Berlin, 153 Teltow-Seehof, Fernruf: Teltow 4831; Prof. Dr.-Ing. habil. Wolfgang Bobeth, Institut für Technologie der Fasern der Deutschen Akademie der Wissenschaften zu Berlin, 801 Dresden, Hohe Str. 6, Fernruf: 44721; Prof. Dr.-Ing. Hans Bōhringer, Institut für Textiltechnologie der Chemiefasern Rudolder Deutschen Akademie der Wissenschaften zu Berlin, 153 Teltow-Seehof, Fernruf: Teltow 4831 . Schriftleiter: Joachim Brämer und Dipl.-Chem. Ingebor Ruscher, 153 Teltow-Seehof, Kantstr. 55. Verlag: Akademie-Verlag GmbH., 108 Berlin, Leipziger Str. 3-4. Satz und Druck: Druckhaus „Maxim Gorki“" 74 Altenburg. - Veróffentlicht unter der Lizenzn ummer 1280 des Presseamtes beim Vorsitzenden des Ministerrates der Deutschen Demokratischen Republik.

Manuskriptsendungen sind an einen der Herausgeber oder die Schriftleitung zu richten. Für Inhalt und Form gelten die „Richtlinien für die Annahme und 50 Sonderdrucke ihrer Arbeit unentgeltlich. Nachdrucke sowie . Die Verfasser größerer wissenschaftlicher Arbeiten erhalten außer dem Honorar ein Heft un auszugsweise mit Quellenangabe - bedürfen der schriftlichen Vereinbarung mit dem Verlag. 